\title{
Surface Modification and Characterization of Coconut Shell-Based Activated Carbon Subjected to Acidic and Alkaline Treatments
}

\author{
Tan, I. A. W. ${ }^{a}{ }^{*}$, Abdullah, M. O. ${ }^{a}$, Lim, L. L. P. ${ }^{\mathrm{b}}$, Yeo, T. H. C. ${ }^{\mathrm{a}}$ \\ ${ }^{a}$ Department of Chemical Engineering and Energy Sustainability, Universiti Malaysia \\ Sarawak, 94300 Kota Samarahan, Malaysia. \\ ${ }^{b}$ Department of Civil Engineering, Universiti Malaysia Sarawak, 94300 Kota Samarahan, \\ Malaysia.
}

\begin{abstract}
Activated carbon derived from agricultural biomass has been increasingly recognized as a multifunctional material for various applications according to its physicochemical characteristics. The application of activated carbon in adsorption process mainly depends on the surface chemistry and pore structure which is greatly influenced by the treatment method. This study aims to compare the textural characteristics, surface chemistry and surface morphology of coconut shell-based activated carbon modified using chemical surface treatments with hydrochloric acid ( $\mathrm{HCl})$ and sodium hydroxide $(\mathrm{NaOH})$. The untreated and treated activated carbons were characterized for their physical and chemical properties including the Fourier transform infrared (FTIR) spectroscopy, scanning electron microscopy (SEM) and textural characterization. The FTIR spectra displayed bands confirming the presence of carboxyl, hydroxyl and carbonyl functional groups. The BrunauerEmmett-Teller (BET) surface area of the untreated activated carbon was $436 \mathrm{~m}^{2} / \mathrm{g}$ whereas the surface area of the activated carbon modified using $1 \mathrm{M} \mathrm{NaOH}, 1 \mathrm{M} \mathrm{HCl}$ and $2 \mathrm{M} \mathrm{HCl}$ was 346, 525 and $372 \mathrm{~m}^{2} / \mathrm{g}$, respectively. SEM micrographs showed that many large pores in a honeycomb shape were clearly found on the surface of $1 \mathrm{M} \mathrm{HCl}$ sample. The pore structure of the activated carbon treated with $2 \mathrm{M} \mathrm{HCl}$ and $\mathrm{NaOH}$ was partially destroyed or enlarged, which decreased the BET surface area. The modification of the coconut shell-based activated carbon with acidic and alkaline treatments has successfully altered the surface functional groups, surface morphology and textural properties of the activated carbon which could improve its adsorptive selectivity on a certain adsorbate.
\end{abstract}

Keywords: Activated carbon, Surface modification, Surface characteristics, Surface morphology.

\section{Introduction}

Activated carbon is the most commonly used adsorbent in separation and purification processes due to its large adsorptive capacity. Activated carbon has been proven effective in removing a wide variety of organic and inorganic pollutants dissolved in aqueous media or from gaseous environment [1-5]. Nevertheless, the wide use of commercial activated carbon conventionally derived from non-renewable precursors such as coal, is limited by the unjustified application for pollution

\footnotetext{
* Corresponding author. Tel.: +6082-583312; fax: +6082-583410

E-mail address: awitan@unimas.my

Manuscript History:

Received 25 May, 2017, Revised 17 July, 2017, Accepted 31 July, 2017, Published 30 September, 2017
}

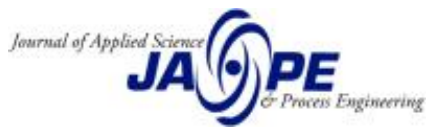


control particularly in terms of cost and long term availability of material. This has prompted a growing research interest in the production of activated carbon from renewable and cheaper precursors [6]. However, it is important to understand the various factors influencing the adsorption capacity of the activated carbon prior to the modification so that it can be tailored to the specific physical and chemical characteristics in order to enhance the affinities towards metal, inorganic or organic species. These factors include specific surface area, pore size distribution, pore volume and surface chemistry [7]. The texture of activated carbon may be adapted to suit the situation by adequate choice of the activation procedure; hence it is possible to prepare activated carbon with different proportions of micropores, mesopores and macropores. Carboxyl, carbonyl, phenol, quinone and lactone groups have been generally identified on activated carbon surfaces [1]. The nature and concentration of the surface functional groups may be modified by suitable thermal or chemical post-treatments. The concentration of the surface oxygen groups can be increased by oxidation in the gas or liquid phase while some of these groups can be selectively removed by heating under inert atmosphere [8]. Acidic treatment of activated carbon is generally employed to oxidize the porous carbon surface as it increases the acidic property, removes the mineral elements and improves the hydrophilic nature of carbon surface [9]. Alkaline treatment of activated carbon produces positive surface charge which in turn is helpful to adsorb negatively charged species in higher amount [1].

The adsorption capacity of activated carbon is determined by the porous structure whereas the interaction with specific or polar adsorbates is determined by the surface functional group [10]. The adsorption capacity of activated carbon can also be identified from its textural characteristics such as porosity, surface area and pore size [11]. Both microporous structure and functional group have been reported to influence the extent of adsorption and reduction processes. The surface treatment of the activated carbon with acidic or alkaline solutions was performed in a study by Park and Jang [10] to give functional groups, which improved the adsorption capacity and selectivity on a certain adsorbate in the gaseous and liquid phase. Chun et al. [7] found that the oxidative treatment of activated carbon was very favourable for enhancing uptakes of metal ions, while thermal treatment of activated carbon was generally favourable for enhancing adsorption of organic compounds from aqueous solutions, since alkaline characteristics of activated carbon was amplified at high temperature condition.

The presence of surface functional groups influences the acidic, alkaline or neutral characteristic of the activated carbon. The acidic functional group on activated carbon surface can be increased via modification of activated carbon with acid. Moreover, it also enhances the chelation ability with metal species; however it may decrease the specific surface area, pore volume and has adverse effect on the uptake of organics. On the other hand, modification of activated carbon with alkaline can enhance the uptake of organics but it may, in some cases, decrease the uptake of metal ions [7]. The activated carbon treated with hydrochloric acid showed that the Brunauer-EmmettTeller (BET) surface area increased by $4.93 \%$ [10]. The results showed that acidic treatment of activated carbon had beneficial effects on adsorption of metal ions. In most cases, treatment with oxidizing agent alters the surface chemistry of the original activated carbon as well as affecting the textural characteristics of the original activated carbon, either increasing or decreasing the specific surface area, pore volume and pore diameter. The focus of this research is to compare the textural characteristics, surface chemistry and surface morphology of coconut-shell based activated carbon modified using chemical surface treatments with hydrochloric acid $(\mathrm{HCl})$ and sodium hydroxide $(\mathrm{NaOH})$. The characterization of the activated carbon was performed respectively in terms of nitrogen gas adsorption isotherm, Fourier transform infrared (FTIR) spectroscopy and scanning electron microscopy (SEM). 


\section{Materials and methods}

\subsection{Pre-treatment of activated carbon}

Granular activated carbon derived from coconut shell was used as the precursor in this investigation. The activated carbon sample was washed with distilled water and dried at $80{ }^{\circ} \mathrm{C}$ for 24 hours before the modification process was carried out. The dried sample was then subjected to chemical modification by using $\mathrm{HCl}$ and $\mathrm{NaOH}$.

\subsection{Preparation of acidic and alkaline solutions}

Concentrated $\mathrm{HCl}$ solution (37\%) from Merck and $\mathrm{NaOH}$ solution (50\%) from Sigma-Aldrich were used as the chemical modification agents whereas acetone from Merck was used for rinsing the apparatus during modification and FTIR characterization. The $\mathrm{HCl}$ solution was diluted to $1 \mathrm{M}$ and $2 \mathrm{M}$, whereas the $\mathrm{NaOH}$ solution was diluted to $1 \mathrm{M}$, by using deionized water.

\subsection{Preparation of HCl-treated activated carbon}

For acidic treatment, the activated carbon was modified by soaking $2 \mathrm{~g}$ of the activated carbon in $50 \mathrm{ml}$ of $1 \mathrm{M}$ and $2 \mathrm{M} \mathrm{HCl}$ solutions, respectively. The samples were denoted as $1 \mathrm{HCl}$ and $2 \mathrm{HCl}$, respectively. The samples were left for 24 hours at room temperature $\left(28 \pm 2^{\circ} \mathrm{C}\right)$. Then, the samples were filtered with filter paper (Whatman No.1) to separate the activated carbon from the solution and then dried overnight at $80{ }^{\circ} \mathrm{C}$ in an oven to constant weight.

\subsection{Preparation of $\mathrm{NaOH}-$ treated activated carbon}

Alkaline treatment of the activated carbon was done by soaking $2 \mathrm{~g}$ of the activated carbon in 50 $\mathrm{ml}$ of $1 \mathrm{M} \mathrm{NaOH}$, and left for 24 hours at room temperature $\left(28 \pm 2^{\circ} \mathrm{C}\right)$. The sample was denoted as $1 \mathrm{NaOH}$, and was similarly filtered with filter paper (Whatman No.1) and dried overnight at $80{ }^{\circ} \mathrm{C}$ in an oven.

\subsection{Characterization of activated carbon}

The chemically modified activated carbons were characterized for their physical and chemical properties by using FTIR, SEM and textural characterization.

\subsubsection{FTIR characterization}

The surface functional groups of the untreated activated carbon and the modified activated carbons were detected by using FTIR spectrophotometer (Perkin Elmer Spectrum GX). The solid samples were prepared using potassium bromide $(\mathrm{KBr})$ as diluents in which the activated carbons were prepared as a thin layer of disc. The sample was ground together with $\mathrm{KBr}$ in the ratio of 1:100. It was used to characterize the entire molecules by simple injection and as the reference to generalize the chart of characteristic group frequency. The spectra were recorded from 4000 to $400 \mathrm{~cm}^{-1}$.

\subsubsection{SEM characterization}

SEM was used to study the surface morphology of the activated carbon. The morphology was studied at an accelerating voltage of $10 \mathrm{kV}$. The activated carbon particles were attached on an 
aluminum stub by using double-sided tape and were covered by a thin layer of platinum coating by using a coater. The samples were placed in SEM instrument (JEOL JSM-6390LA) with magnification of $500 \mathrm{X}$.

\subsubsection{Textural characterization}

The specific surface area of the activated carbon was measured by the BET method using nitrogen gas adsorption at $77 \mathrm{~K}$ by using a nitrogen adsorption/desorption analyzer (Thermo-Finnigan Sorptomatic 1990 SERIES). The specific surface area was calculated using Langmuir equation whereas the total volume was estimated from the nitrogen gas uptake.

\section{Results and discussion}

\subsection{FTIR characterization}

The chemical reactivity of the surface especially in the form of chemisorbed oxygen in various forms of functional group influences the adsorptive behaviour of activated carbon. These surface oxides have acidic as well as base properties [11]. The FTIR spectra for the activated carbons treated using $1 \mathrm{M} \mathrm{HCl}, 2 \mathrm{M} \mathrm{HCl}$ and $1 \mathrm{M} \mathrm{NaOH}$ are shown in Figure 1.

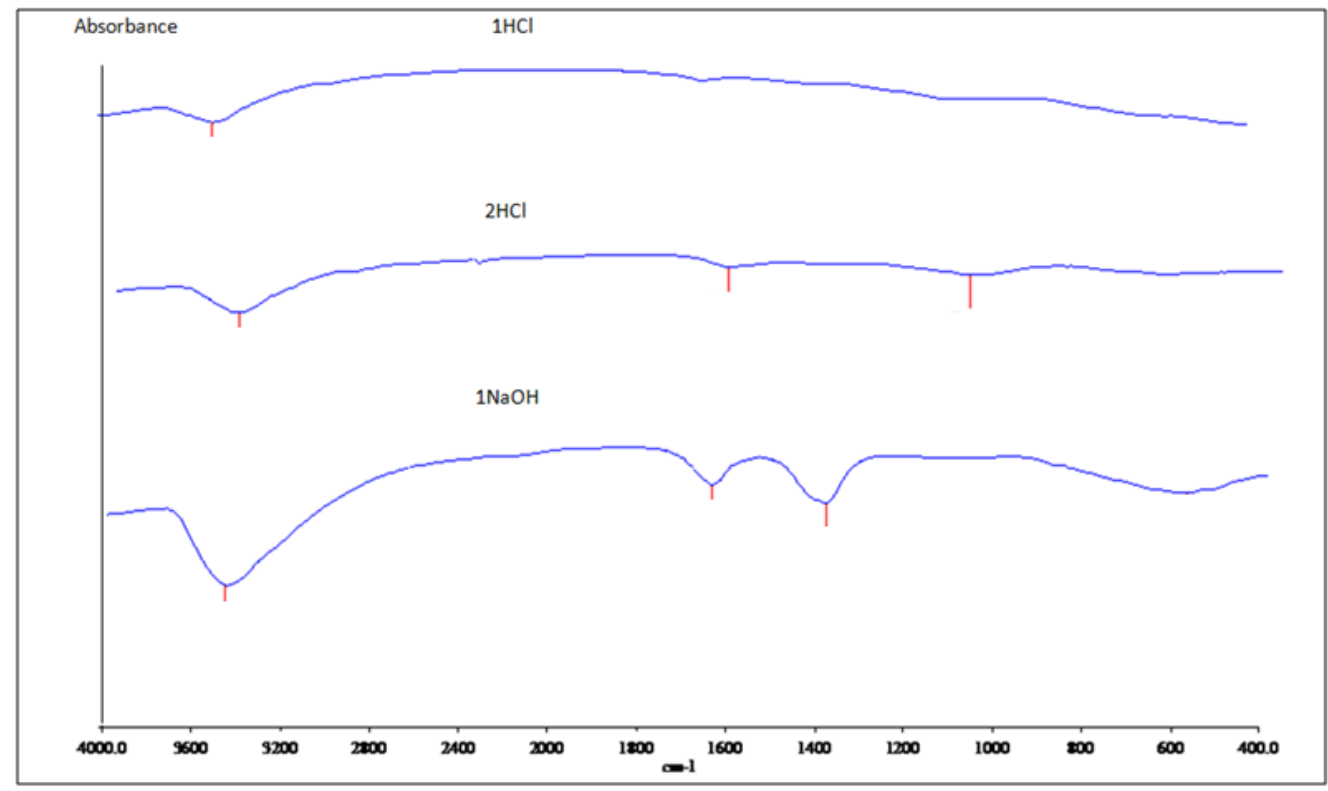

Figure 1. FTIR spectra for activated carbons treated using $1 \mathrm{M} \mathrm{HCl}, 2 \mathrm{M} \mathrm{HCl}$ and $1 \mathrm{M} \mathrm{NaOH}$

The spectrum showed that all the unmodified and modified activated carbons had almost similar infrared spectra absorption band between the regions of $3200-3600 \mathrm{~cm}^{-1}$. The band found in unmodified activated carbon in the region of $3400 \mathrm{~cm}^{-1}$ was a result of an $\mathrm{O}-\mathrm{H}$ stretching mode of hydroxyl group in alcohols, phenols or absorbed water [12]. This peak was slightly broader in the modified samples in which the band slightly shifted to $3450-3500 \mathrm{~cm}^{-1}$. As compared to $1 \mathrm{HCl}$ and $2 \mathrm{HCl}$ samples, the peak was more pronounced in $1 \mathrm{NaOH}$ sample which would imply the presence of more O-H group from the increased carboxylic group. The absorption peak near $1700 \mathrm{~cm}^{-1}$ for $1 \mathrm{NaOH}$ sample indicated the presence of carboxyl group. This was in agreement with the findings by Shim et al. [13] in which the presence of the band indicated that there were still carboxyl structures in the micropores which were inaccessible to the base, or $\mathrm{C}=\mathrm{O}$ group which was not neutralized by $\mathrm{NaOH}$ 
solution. The absorption band at $1410 \mathrm{~cm}^{-1}$ increased due to the lactone structure after acidic treatment [14]. The C-O group was shown by the absorption peak at $1100 \mathrm{~cm}^{-1}$ for $2 \mathrm{HCl}$ sample. Jia and Thomas [15] reported the incorporation of acidic oxygen functional groups into activated carbon by $\mathrm{HNO}_{3}$ oxidation and studied the potential of modified carbon towards cadmium removal. It was observed that carboxylic acid groups were the major surface species incorporated, and phenol and quinone groups were introduced during the oxidation process. Chiang et al. [16] found that the activated carbon treated with $\mathrm{NaOH}$ showed major increase in concentration of phenolic functional groups on the surface.

\subsection{SEM characterization}

Figures 2(a)-(d) show the SEM micrographs of the unmodified and modified activated carbons, respectively. The principal morphology of the activated carbon derived from coconut shell was shown by the small pores on the surface. Many small pores in a honeycomb shape were observed on the surface of the activated carbon after the treatment using $1 \mathrm{M} \mathrm{HCl}$. This indicated that $1 \mathrm{M} \mathrm{HCl}$ effectively created well-developed pores on the surface of the activated carbon. However, the treatment using higher concentration of $2 \mathrm{M} \mathrm{HCl}$ resulted in the inconsistent pore structure on the surface of the activated carbon where the honeycomb structure, as shown in $1 \mathrm{HCl}$ sample, either disappeared or collapsed, hence leading to smaller surface area. Li et al. [17] found that the pore structure of coconut shell-based activated carbon treated with acids of high concentration was partially destroyed or enlarged, which greatly decreased the BET surface area. Figure 2(d) shows that the pores on the activated carbon were enlarged after treatment using $1 \mathrm{M} \mathrm{NaOH}$, which also resulted in the smaller surface area. The results were overall in agreement with the work of Park and Jang [10] where the treatment using $\mathrm{NaOH}$ on activated carbon was found to reduce the surface area and micropore volume. Babatunde et al. [12] reported that the activation stage produced an extensive external surface on the coconut shell-derived activated carbon with quite a number of irregular cracks and pores, whereas larger and well-developed pores were clearly found on the surface of the chemically modified sample.
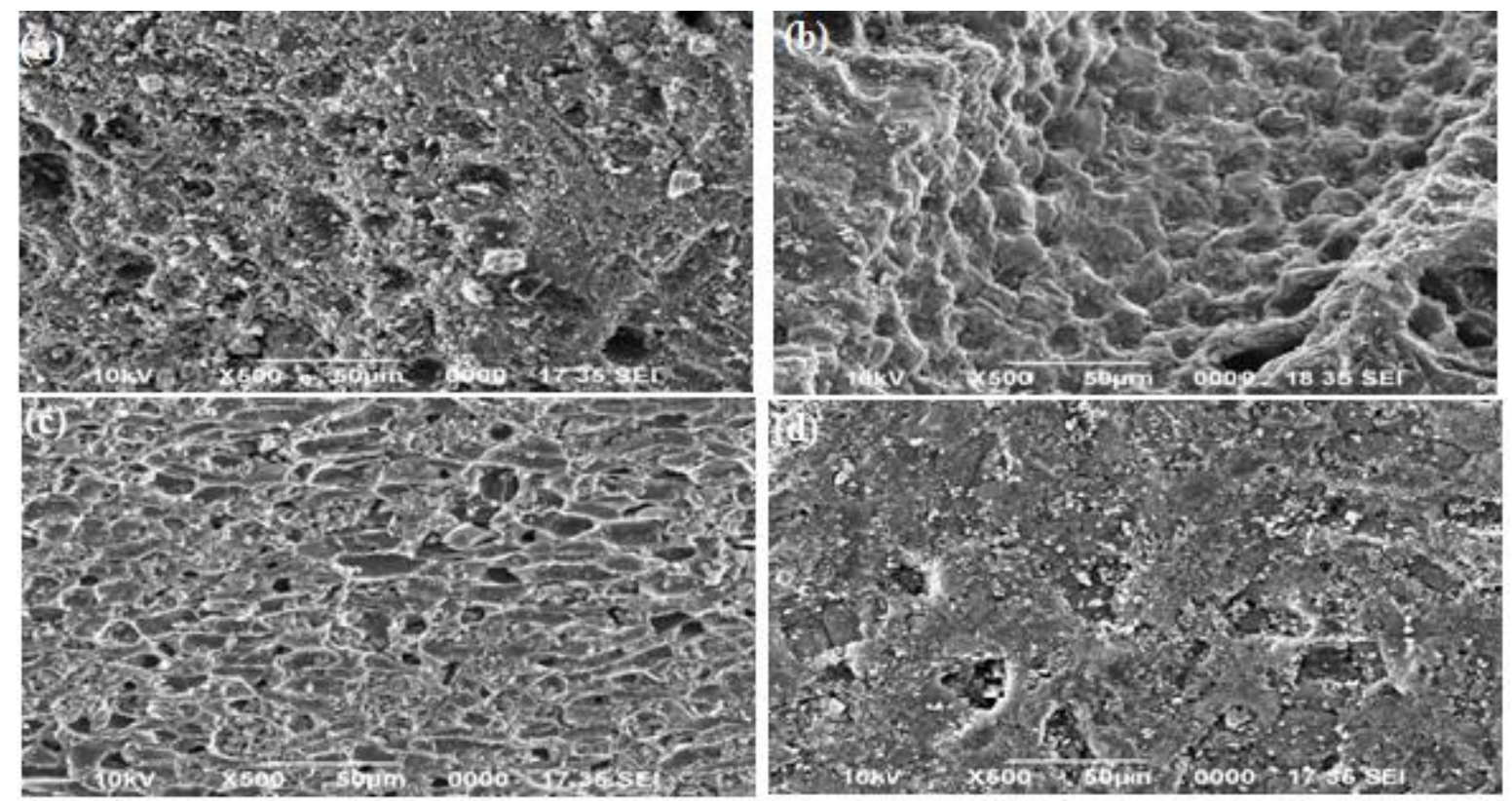

Figure 2. SEM images of (a) untreated activated carbon, (b) $1 \mathrm{M} \mathrm{HCl}$, (c) $2 \mathrm{M} \mathrm{HCl}$ and (d) $1 \mathrm{M} \mathrm{NaOH}$. 


\subsection{Textural characterization}

The textural characteristics of the activated carbons are summarized in Table 1 which include the BET surface area, micropore volume, total pore volume and average pore diameter. The BET surface area of the untreated activated carbon, $1 \mathrm{HCl}, 2 \mathrm{HCl}$ and $1 \mathrm{NaOH}$ were $436,525,372$ and 346 $\mathrm{m}^{2} / \mathrm{g}$, respectively. This indicated that the treatment using $1 \mathrm{M} \mathrm{HCl}$ increased the BET surface area by $20 \%$, as compared to the untreated activated carbon. The BET surface area of $2 \mathrm{HCl}$ sample was lower than that of untreated activated carbon and $1 \mathrm{HCl}$ sample. The reduction of surface area at higher $\mathrm{HCl}$ concentration was possibly due to the destruction effect resulted from the surplus water vapor released via acid dehydration [18]. Similar phenomenon was observed on $1 \mathrm{NaOH}$ sample where the BET surface area decreased after the treatment. This was in agreement with the findings reported by Shim et al. [13] which stated that the decrease of surface area was mainly ascribed to the decrease of the micropore volume. A commercial activated carbon, Chemviron F400, had been modified via oxidation with nitric acid to introduce a variety of acidic surface functional groups on the carbon surface by Vladimir and Malik [19]. The results revealed that the surface area and pore volume were reduced after the oxidation treatment. According to Babatunde et al. [12], the modification of activated carbon using nitric acid produced better physical properties and higher iodine adsorption than $\mathrm{NaOH}$. The average pore diameter of the untreated activated carbon, $1 \mathrm{HCl}, 2 \mathrm{HCl}$ and $1 \mathrm{NaOH}$ were $14.27,14.20$, 12.43 and $12.56 \AA$, respectively. The pores within porous solids are normally classified into micropores $(<2 \mathrm{~nm}$ diameter), mesopores $(2-50 \mathrm{~nm})$ and macropores $(>50 \mathrm{~nm})$ [20]. Hence, the average pore diameter of the activated carbons obtained in this work suggested a predominant micropore.

Table 1. Textural characteristics of activated carbons before and after chemical treatment

\begin{tabular}{lllll}
\hline & Untreated & 1HCl & 2HCl & 1NaOH \\
\hline BET surface area $\left(\mathrm{m}^{2} / \mathrm{g}\right)$ & 436 & 525 & 372 & 346 \\
Micropore volume $\left(\mathrm{cm}^{3} / \mathrm{g}\right)$ & 0.218 & 0.272 & 0.200 & 0.186 \\
Total pore volume $\left(\mathrm{cm}^{3} / \mathrm{g}\right)$ & 0.229 & 0.291 & 0.229 & 0.199 \\
Average pore diameter $(\AA)$ & 14.27 & 14.20 & 12.43 & 12.56 \\
\hline
\end{tabular}

\subsection{Adsorption isotherm}

Adsorption isotherm indicates the distribution of the adsorption molecules when the process reaches equilibrium state between the liquid phase and the solid phase. The analysis of the isotherm data by fitting them to different isotherm models is an important step to find the suitable model that can be used for design purposes [21]. The nitrogen adsorption-desorption isotherms of the untreated and modified activated carbons are shown in Figure 3. The adsorption-desorption isotherms were approximately Type I according to the BET classification [22]. In this work, the isotherm and porosities of the activated carbons treated using $2 \mathrm{M} \mathrm{HCl}$ and $1 \mathrm{M} \mathrm{NaOH}$ indicated that some of the pores were blocked by oxide functional groups introduced by the chemical treatments. The decrease in total pore volume of $0.030 \mathrm{~cm}^{3} / \mathrm{g}$ was concurrent with the decrease in micropore volume of 0.032 $\mathrm{cm}^{3} / \mathrm{g}$ after the chemical modification by using $\mathrm{NaOH}$. Most of the pore volumes of the activated carbon samples were filled below relative pressure about 0.1 , indicating that these activated carbons were highly microporous [8].

Figures 4(a)-(d) show the Langmuir isotherms of the untreated and modified activated carbons, respectively. The $\mathrm{R}^{2}$ values obtained from the isotherms for all the activated carbons were above 0.99 , indicating that the adsorption data were well fitted into the Langmuir isotherm model. 


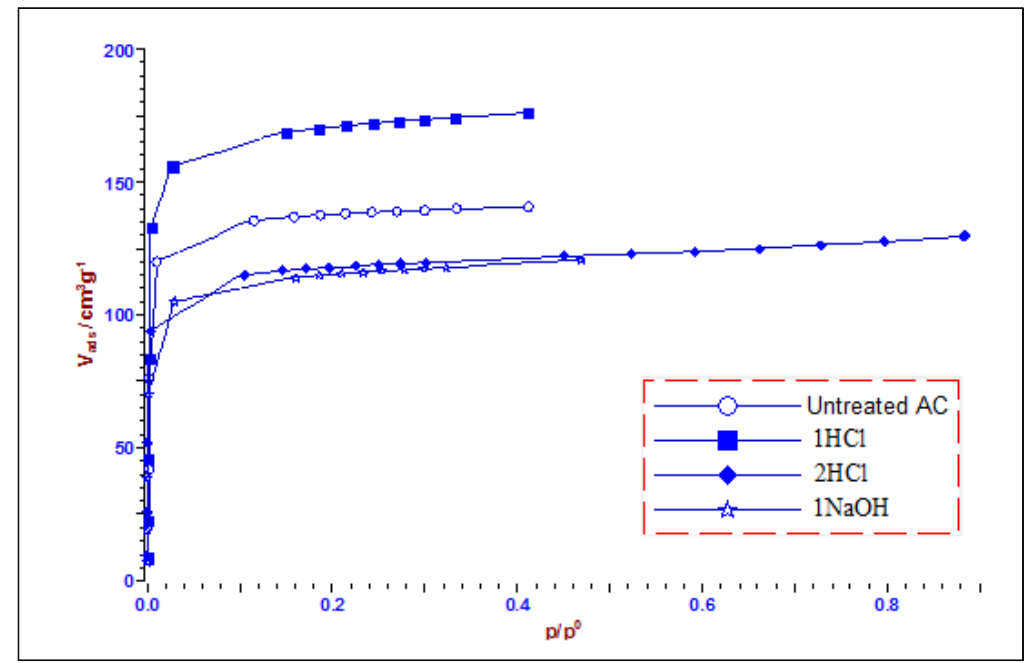

Figure 3. Nitrogen adsorption-desorption isotherms of untreated and modified activated carbons.
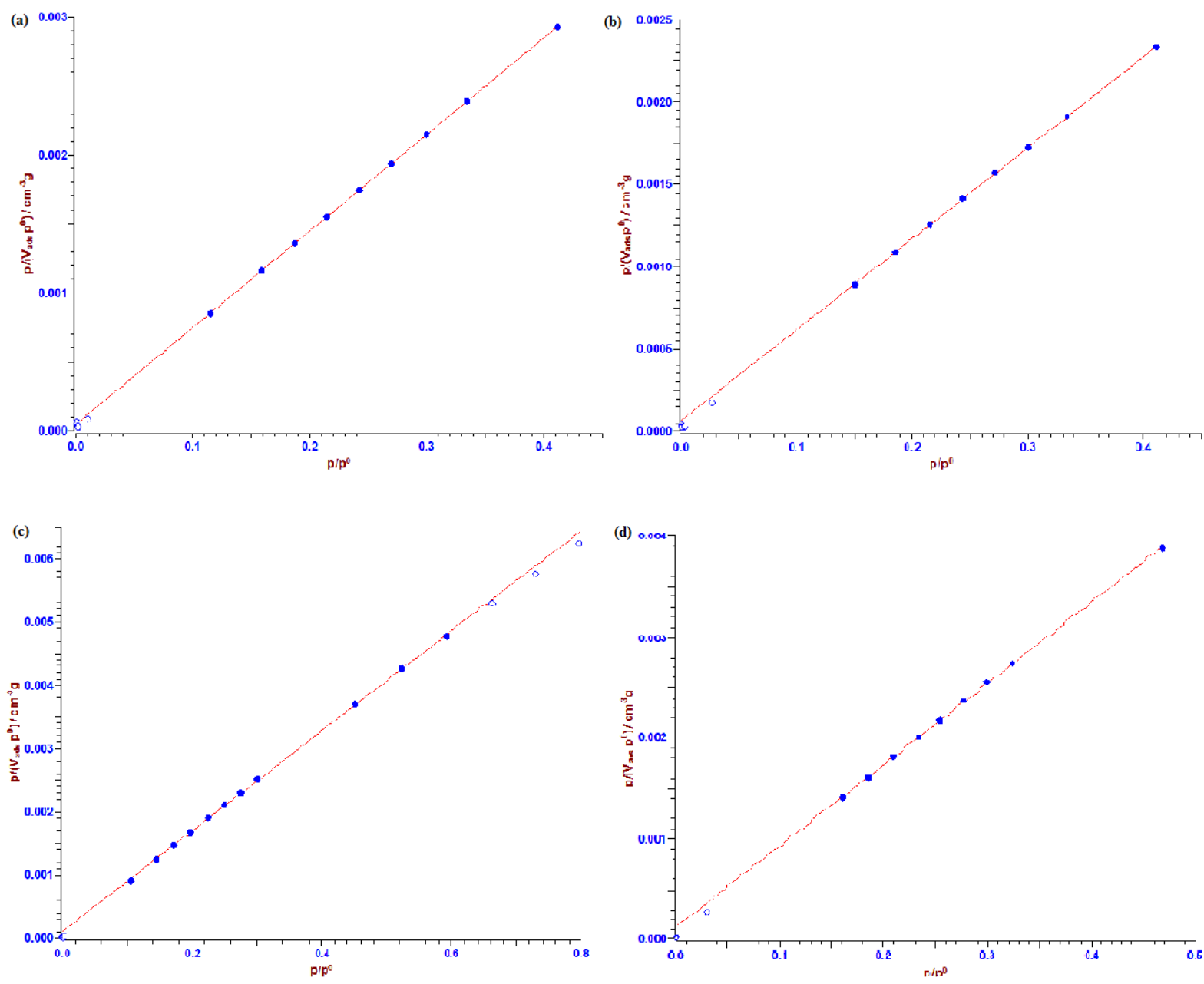

Figure 4. Langmuir isotherms for (a) untreated activated carbon, (b) $1 \mathrm{M} \mathrm{HCl}$, (c) $2 \mathrm{M} \mathrm{HCl}$ and (d) $1 \mathrm{M}$ $\mathrm{NaOH}$. 


\section{Conclusion}

The FTIR spectra displayed bands confirming the presence of carboxyl, hydroxyl and carbonyl functional groups. The treatment using $1 \mathrm{M} \mathrm{HCl}$ created well-developed pores on the surface of the activated carbon. The BET surface area of the activated carbon was increased by $20 \%$ with the modification using $1 \mathrm{M} \mathrm{HCl}$. The micropore volume and total pore volume were also increased by $24 \%$ and $27 \%$, respectively after the chemical treatment. The micropore volume and the total pore volume were however decreased by $15 \%$ and $13 \%$, respectively for the activated carbon modified using $1 \mathrm{M} \mathrm{NaOH}$. The average pore diameter of the activated carbon obtained in this work suggested a predominant micropore. The $\mathrm{R}^{2}$ values obtained for all the unmodified and modified activated carbons were above 0.99 , indicating that the adsorption data were well-fitted into the Langmuir isotherm model. The modification of the coconut shell-based activated carbon with acidic and alkaline treatments has successfully altered the surface functional groups, surface morphology and textural properties of the activated carbon which could improve the adsorptive selectivity on a certain adsorbate.

\section{Acknowledgements}

The authors acknowledge the research grant provided by Universiti Malaysia Sarawak under Special Grant Scheme F02/SpGS/1406/16/7.

\section{References}

[1] Bhatnagar, A., Hogland, W., Marques, M. and Sillanpaa, M. (2013). An overview of the modification methods of activated carbon for its water treatment applications, Chem. Eng. J. 219, 499-511.

[2] Botomé, M.L., Poletto, P., Junges, J., Perondi, D., Dettmer, A. and Godinho, M. (2017). Preparation and characterization of a metal-rich activated carbon from CCA-treated wood for $\mathrm{CO}_{2}$ capture, Chem. Eng. J. 321, 614-621.

[3] Björklund, K. and Li, L.Y. (2017). Adsorption of organic stormwater pollutants onto activated carbon from sewage sludge, J. Environ. Manage. 197, 490-497.

[4] Kumar, A. and Jena, H.M. (2017). Adsorption of Cr(VI) from aqueous phase by high surface area activated carbon prepared by chemical activation with $\mathrm{ZnCl}_{2}$, Process Safety Environ. Protection 109, 63-71.

[5] Ncibi, M.C. and Sillanpää, M. (2017). Optimizing the removal of pharmaceutical drugs Carbamazepine and Dorzolamide from aqueous solutions using mesoporous activated carbons and multi-walled carbon nanotubes, J. Molecular Liquids 238, 379-388.

[6] Tan, I.A.W., Ahmad, A.L. and Hameed, B.H. (2008). Adsorption of basic dye on high-surface-area activated carbon prepared from coconut husk: Equilibrium, kinetic and thermodynamic studies, J. Hazard. Mater. 154, 337-346.

[7] Chun, Y.Y., Aroua, M.K. and Daud, W.M.A.W. (2007). Review of modification of activated carbon for enhancing contaminant uptakes from aqueous solutions, Sep. Purif. Technol. 52, 403-415.

[8] Figueredo, J.L., Pereira, M.F.R., Freitas, M.M.A. and O'rfao, J.J.M.O. (1999). Modification of the surface chemistry of activated carbons, Carbon 37, 1379-1389.

[9] Shen, W., Li, Z. and Liu, Y. (2008). Surface chemical functional groups modification of porous carbon, Recent Patents Chem. Eng. 1, 27-40.

[10] Park, S.J. and Jang, Y.S. (2002). Pore structure and surface properties of chemically modified activated carbons for adsorption mechanism and rate of Cr (IV), J. Colloid Interf. Sci. 249, 458-463.

[11] Ahmad, A.L., Loh, M.M. and Aziz, J.A. (2007). Preparation and characterization of activated carbon from oil palm wood and its evaluation on methylene blue adsorption, Dyes Pigments 263, 263-272. 
[12] Babatunde, O.A., Garba, S. and Ali, Z. N. (2016). Surface modification of activated carbon for improved iodine and carbon tetrachloride adsorption, Am. J. Chem. 6, 74-79.

[13] Shim, J.W., Park, S.J. and Ryu, S.K. (2001). Effect of modification with $\mathrm{HNO}_{3}$ and $\mathrm{NaOH}$ on metal adsorption by pitch-based activated carbon fibers, Carbon 39, 1635-1642.

[14] Zawadzki, J. (1989). Infrared spectroscopy in surface chemistry of carbons, Chem. and Phys. Carbon 21, 147-380.

[15] Jia, Y.F. and Thomas, K.M. (2000). Adsorption of cadmium ions on oxygen surface sites in activated carbon, Langmuir 16, 1114-1122.

[16] Chiang, H.L., Huang, C.P. and Chiang, P.C. (2002). The surface characteristics of activated carbon as affected by ozone and alkaline treatment, Chemosphere 47, 257-265.

[17] Li, L., Liu, S. and Liu, J. (2011). Surface modification of coconut shell based activated carbon for the improvement of hydrophobic VOC removal, J. Haz. Mater. 192, 683-690.

[18] Yakout, S.M., Daifullah, A.E.H.M. and El-Reefy, S.A. (2015). Pore structure characterization of chemically modified biochar derived from rice straw, Environ. Eng. Manage. J. 14, 473-480.

[19] Vladimir, S.J. and Malik, D. (2002). Characterization and metal sorptive properties of oxidized active carbon, J. Colloid Interf. Sci. 250, 213-220.

[20] Lua, A.C. and Guo, J. (2001). Preparation and characterization of activated carbons from oil-palm stones for gas-phase adsorption, Colloid. Surface. A 179, 151-162.

[21] El-Guendi, M. (1991). Homogeneous surface diffusion model of basic dyestuffs onto natural clay in batch adsorbers, Adsorpt. Sci. Technol. 8, 217-225.

[22] Gregg, S.J. and Sing. K.S.W. (1982). Adsorption, Surface Area and Porosity. London: Academic Press. 